\title{
The Good, Bad, and Volatility Beta: A Generalized CAPM
}

\author{
Ravi Bansal* \\ Dana Kiku ${ }^{\dagger}$ \\ Ivan Shaliastovich $\ddagger$ \\ Amir Yaron ${ }^{\S}$
}

February 15, 2011

\begin{abstract}
In this paper we develop an economic asset pricing framework that identifies three key sources of risk that underlie the risk and return tradeoff in the economy: news to cashflows, news to expected returns, and news to aggregate volatility. A novel contribution of this paper is the inclusion of time-varying volatility news which has both important theoretical and empirical implications. Motivated by a Long Run Risk framework, we theoretically show that to consistently identify and explain the time series and cross sectional variation in risk premia it is essential to allow for time varying economic uncertainty in the model. Empirically, we show that the exposure of assets to volatility risk, sorted by size and book to market, line up vary well with those assets' average excess return in the data. The model's fit of a broad cross section of asset returns is about $90 \%$ of which the volatility news factor contributes $20 \%$. The volatility component plays a significant role in explaining the level of the premium as well as the value and size spreads.
\end{abstract}

*Fuqua School of Business, Duke University and NBER, ravi.bansal@duke.edu.

${ }^{\dagger}$ The Wharton School, University of Pennsylvania, kiku@wharton.upenn.edu.

† The Wharton School, University of Pennsylvania, ishal@wharton.upenn.edu.

$\S$ The Wharton School, University of Pennsylvania and NBER, yaron@wharton.upenn.edu. 